\title{
Atributos químicos de um Neossolo Regolítico sob uso de dejeto de suínos
}

\author{
Chemical attributes of an Entisol under swine manure treatment
}

\section{Patrícia Pretto Pessotto ${ }^{*}$, Rodrigo Ferreira da Silva ${ }^{2}$, Clovis Orlando da Ros ${ }^{2}$, Vanderlei Rodrigues da Silva ${ }^{2}$, Diego Armando Amaro da Silva ${ }^{3}$ \& André Luis Grolli ${ }^{2}$}

\author{
${ }^{1}$ Universidade do Estado de Santa Catarina, Lages, SC, Brasil. *Autor para correspondência: paty.pessotto@hotmail.com. \\ ${ }^{2}$ Universidade Federal de Santa Maria, Frederico Westphalen, RS, Brasil. \\ ${ }^{3}$ Universidade Federal de Lavras, Lavras, MG, Brasil.
}

Submissão: 17/02/2017 | Aceite: 13/06/2018

\begin{abstract}
RESUMO
A distribuição superficial no solo de dejetos líquidos de suínos (DLS) visando a fertilização das culturas é uma atividade comum nas propriedades rurais produtoras de suínos. O objetivo do trabalho foi avaliar a qualidade do solo em função da quantificação dos atributos químicos do solo em diferentes sistemas de manejo e tempo de uso de DLS. Foram avaliados atributos químicos de um Neossolo Regolítico, em três camadas. Os tratamentos foram compostos por uma área de mata nativa (MN) e três áreas de cultivo: pastagem perene de tifton 85 (Cinodon spp.) com uso sucessivo de DLS durante quatro anos (PP4) e 16 anos (PP16) e cultivo anual de grãos em plantio direto durante 16 anos (PD), localizadas em uma propriedade rural do município de Três Passos, RS. O uso de DLS em PP4 e PP16 aumentaram o teor de $\mathrm{Cu}$ e Zn na camada superficial do solo mostrando contribuição significativa dos DLS no aumento destes elementos no solo. A aplicação sucessiva de DLS em PP16 aumentou os teores P na camada de $0-5 \mathrm{~cm}$ acima do limite crítico ambiental (LCA-P), podendo comprometer a qualidade do solo. A capacidade de troca de cátions (CTC), carbono orgânico total (COT) e os teores de $\mathrm{Cu}, \mathrm{Zn}$ e $\mathrm{P}$ no solo são os atributos químicos que podem ser usados como indicadores de qualidade do solo.
\end{abstract}

PALAVRAS-CHAVE: adubação orgânica, manejo do solo, pastagem perene, ambiente.

\begin{abstract}
The surface distribution of liquid pig manure on the soil aiming crop fertilization is a common activity in pig farms. The objective of this study was to evaluate the soil quality according to the quantification of its chemical attributes in different management systems and time of use of liquid pig manure. Chemical attributes of an Entisol were evaluated in three layers. The treatments consisted of a native forest area (NF) and three farming areas: perennial Tifton 85 (Cinodon spp.) pasture with successive use of pig manure for 4 (PP4) and 16 years (PP16) and annual cultivation of grains in no-till farming for 16 years (NTF), located in a rural property in the municipality of Três Passos, Rio Grande do Sul, Brazil. The use pig maure in PP4 and PP16 increased the $\mathrm{Cu}$ and $\mathrm{Zn}$ content in the soil surface layer when compared to the native forest area, indicating significant contribution of pig manure in the increase of these elements in the soil. The successive application of pig manure in PP16 increased the P content in the $0-5 \mathrm{~cm}$ layer above the critical environmental limits (CEL-P), which could the soil quality. The cation exchange capacity (CEC), total organic carbon (TOC) and $\mathrm{Cu}, \mathrm{Zn}$ and $\mathrm{P}$ in soil are the chemical attributes that can be used as soil quality indicators. KEYWORDS: organic fertilization, soil management, perennial pasture, environment.
\end{abstract}

\section{INTRODUÇÃO}

No Brasil, especialmente na Região Sul, a suinocultura é uma atividade típica de pequenas propriedades rurais. Esta atividade gera grandes quantidades de dejetos que são utilizados como adubos orgânicos para adubação de pastagens e culturas anuais.

Dentre esses dejetos, destacam-se os dejetos líquidos de suínos (DLS), os quais podem contribuir para o aumento do rendimento das culturas, redução da aplicação de fertilizantes químicos, e também, melhoria nas condições físico-químicas do solo (ANAMI et al. 2008, CASSOL et al. 2012). A aplicação de DLS é uma alternativa ambientalmente sustentável para descarte desse resíduo podendo substituir os adubos minerais 
e representa grande potencial produtivo (BARBOSA et al. 2009). SARTOR et al. (2012), estudando a aplicação semestral de DLS por seis anos, constataram melhoria da produtividade das culturas de milho, feijão, soja e trigo, com aumento no rendimento de grãos e nos teores $\mathrm{K}, \mathrm{P}, \mathrm{Ca}$ e $\mathrm{Mg}$ no solo, podendo substituir o uso de fertilizante mineral. No entanto, aplicações contínuas poderão ocasionar desequilíbrios de nutrientes no solo e a gravidade do problema dependerá do tempo de aplicação, da composição e da quantidade de DLS aplicado, além do tipo de solo e da capacidade de extração pelas plantas (MAGGl et al. 2013).

Elementos traço como o Cu e o Zn, que são adicionados ao solo via DLS ficam adsorvidos nas camadas superficiais do solo (MOREIRA et al. 2009, TIECHER et al. 2013), por permanecerem ligados ou fazendo parte de compostos orgânicos, e desta forma podem causar contaminação do mesmo e, em alguns casos, afetar o crescimento das plantas. Assim como os elementos traço, as aplicações sucessivas de DLS incrementam as quantidades de $\mathrm{P}$ na camada superficial do solo, o qual pode comprometer a qualidade das águas superficiais, pois o $\mathrm{P}$ é facilmente carregado pelo escoamento superficial (CERETTA et al. 2010, GIROTTO et al. 2010), podendo causar eutrofização dos cursos hídricos, uma vez que o Neossolo é pouco profundo e apresenta valores consideráveis de silte e areia. Assim, a quantidade aplicada de DLS é fator determinante, podendo causar contaminação ambiental do solo e das águas.

Neste contexto, o objetivo do trabalho foi avaliar a qualidade química de um Neossolo em função da quantificação dos atributos químicos do solo em diferentes sistemas de manejo e tempo de uso de DLS.

\section{MATERIAL E MÉTODOS}

O estudo foi realizado em uma propriedade rural no município de Três Passos, localizado no noroeste

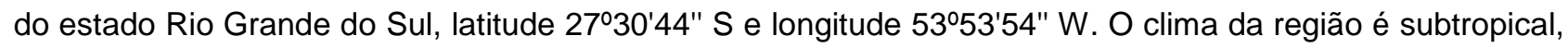
tipo Cfa, segundo a classificação de Köppen, com precipitação pluvial distribuída em todos os meses do ano, com média anual de $1.680 \mathrm{~mm}$ e 18,8 ${ }^{\circ} \mathrm{C}$ (MALUF 2000).

O trabalho foi composto por quatro tratamentos e oito repetições em diferentes usos do solo, sendo uma área de mata nativa ( $\mathrm{MN})$, usada como testemunha, e três áreas de cultivo: 1) pastagem perene de tifton 85 (Cinodon spp.) com uso sucessivo de dejetos líquido de suínos durante quatro anos (PP4); 2) pastagem perene de tifton 85 com uso sucessivo de dejetos líquido de suínos durante 16 anos (PP16); 3) cultivo anual de grãos em plantio direto durante 16 anos (PD), sob adubação mineral. Estas áreas apresentaram 1,50; 0,69; 0,79 e 7,19 ha, respectivamente, situadas em uma distância de no máximo $500 \mathrm{~m}$.

A área de MN foi caracterizada como bioma Mata Atlântica, sem perturbação do ambiente desde 1960. As duas áreas de tifton (PP4 e PP16) receberam, no processo de implantação da pastagem, $2 \mathrm{Mg} \mathrm{ha}^{-1}$ de calcário e são manejadas em sistema de pastejo rotativo com vacas leiteiras e recebem anualmente cerca $150 \mathrm{~m}^{3}$ ha-1 de DLS, dividido em quatro aplicações a lanço, realizadas após o pastejo dos animais. No período outonal foi utilizada a sobressemeadura de azevém devido à diminuição na intensidade de rebrote do tifton. A área em PD caracterizou-se por produção de grãos com adubação mineral (NPK) nas culturas de soja/milho no verão e trigo/aveia preta no inverno.

O solo do local foi classificado como Neossolo Regolítico, com declive ondulado a fortemente ondulado (SANTOS et al. 2013). Na Tabela 1 estão especificadas a profundidade efetiva do perfil do solo, obtida pela diferença de altura entre a superfície do solo e a camada rochosa; a granulometria (teor de argila, areia grosa,

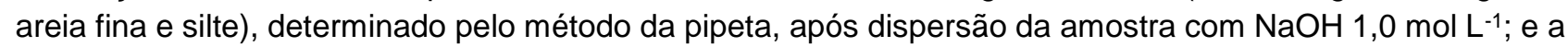
densidade das partículas, quantificada pelo método do balão volumétrico (DONAGEMA et al. 2011), na camada de 0-10 cm, nas quatro áreas.

A coleta de amostras de solo para análise dos atributos químicos foi realizada no mês de outubro de 2014, com pá de corte, nas camadas de 0-5, 5-10 e 10-20 cm. Após secagem e moagem das amostras foram determinados o $\mathrm{pH}$ em água $\left(\mathrm{pH}_{\mathrm{H} 2 \mathrm{O}}\right.$ ) na relação 1:1; carbono orgânico total (COT), pelo método proposto por YEOMANS \& BREMNER (1988); fósforo $(\mathrm{P})$, potássio $(\mathrm{K})$ e sódio $(\mathrm{Na})$ obtidos pelo método de extração Mehlich ${ }^{-1}$; cobre $(\mathrm{Cu})$ e zinco $(\mathrm{Zn})$ pelo método de extração em $\mathrm{HCl}$; cálcio $(\mathrm{Ca})$, magnésio $(\mathrm{Mg})$ trocáveis pelo método de extração em $\mathrm{KCl}$; acidez trocável $\left(\mathrm{Al}^{3+}\right)$; acidez potencial $(\mathrm{Al}+\mathrm{H})$, estimada pelo $\mathrm{pH}$ índice SMP (TEDESCO et al. 1995); e ainda foram calculadas a capacidade de troca de catiônica (CTC) efetiva (CTC efetiva), e potencial $\left(\mathrm{CTC}_{\mathrm{pH}} \mathrm{O}, \mathrm{)}\right)$ e a saturação por bases $(\mathrm{V} \%)$.

Os dados foram submetidos à análise da variância pelo teste $\mathrm{F}$ usando o modelo de delineamento experimental inteiramente casualizado, no esquema fatorial $(4 \times 3)$. As médias de tratamentos foram comparadas pelo teste t de Student, utilizado o programa estatístico SISVAR, tomando como base os níveis de significância maiores que $95 \%(p \leq 0,05)$. 


\section{RESULTADOS E DISCUSSÃO}

A análise da variância evidenciou interação significativa entre os sistemas de manejo e as camadas de solo para a saturação por bases e teores de $\mathrm{COT}, \mathrm{Al}+\mathrm{H}, \mathrm{P}, \mathrm{Ca}, \mathrm{Cu}$ e $\mathrm{Zn}$, e interação não significativa, para o pH, CTC e teores de K, Mg e $\mathrm{Na}$ (Tabela 2).

Tabela 1. Profundidade efetiva do perfil do solo, teor de argila total, areia fina, areia grossa, silte e densidade de partícula na camada de $0-10 \mathrm{~cm}$ nos diferentes sistemas de manejo.

Table 1. Effective depth of soil profile, total clay content, fine sand, coarse sand, silt and particle density in the $0-10 \mathrm{~cm}$ layer in the different management systems.

\begin{tabular}{|c|c|c|c|c|c|c|}
\hline $\begin{array}{l}\text { Sistema de } \\
\text { manejo (1) }\end{array}$ & $\begin{array}{l}\text { Profundidade } \\
\text { efetiva }\end{array}$ & $\begin{array}{r}\text { Argila } \\
\text { total }\end{array}$ & $\begin{array}{c}\text { Areia } \\
\text { fina }\end{array}$ & $\begin{array}{c}\text { Areia } \\
\text { grossa }\end{array}$ & Silte & $\begin{array}{c}\text { Densidade } \\
\text { partícula }\end{array}$ \\
\hline & $(\mathrm{m})$ & \multicolumn{4}{|c|}{----------- g kg$^{-1}$} & $\left(\mathrm{Mg} \mathrm{m}^{-3}\right)$ \\
\hline PP4 & 0,79 & 207,7 & 123,1 & 152,7 & 516,4 & 2,78 \\
\hline PP16 & 0,59 & 300,7 & 102,8 & 123,3 & 473,2 & 2,82 \\
\hline PD & 0,75 & 433,0 & 67,4 & 97,9 & 401,7 & 2,90 \\
\hline $\mathrm{MN}$ & 0,53 & 232,0 & 123,8 & 152,8 & 491,4 & 2,58 \\
\hline
\end{tabular}

(1) PP4: pastagem perene de tifton com uso sucessivo de dejetos líquido de suínos durante quatro anos; PP16: pastagem perene de tifton com uso sucessivo de dejetos líquido de suínos durante 16 anos; PD: cultivo anual de grãos em plantio direto durante 16 anos; MN: mata nativa.

Os teores de Cu disponíveis foram significativamente maiores nas áreas de PD, PP4 e PP16 em relação à $M N$ (Figura 1A). Com o uso de DLS nas duas áreas de cultivo de tifton (PP4 e PP16) houve maior concentração de Cu na camada superficial do solo $(0-5 \mathrm{~cm})$, mas sem diferença entre o tempo de aplicação. Nesta camada, o incremento médio de $\mathrm{Cu}$ foi de 12,8 vezes em relação a $\mathrm{MN}$, indicando contribuição significativa dos dejetos no aumento deste micronutriente no solo. $\mathrm{Na}$ área de PD o incremento de $\mathrm{Cu}$ em relação à $M N$ foi de 9,1 vezes, confirmando que o uso de fertilizante mineral nas culturas anuais durante 16 anos sem revolvimento do solo, aliado ao acumulo de resíduos vegetais sobre o solo contribuíram para o aumento nos teores de $\mathrm{Cu}$ (STEINER et al. 2011), mas em proporção menor em relação à adubação com DLS. Os resultados da distribuição dos teores de Cu nas diferentes camadas de solo e das suas respectivas quantidades nos tratamentos com DLS e adubação mineral foram semelhantes ao trabalho de SCHERER et al. (2010) em Neossolo com culturas anuais em áreas de uso de DLS por um período de 15 e 25 anos e de adubação mineral por 20 anos.

Tabela 2. Probabilidade do teste $F$ da análise da variância e o coeficiente de variação (CV) dos atributos químicos do solo para os quatro sistemas de manejo e nas três camadas de solo.

Table 2. Probability $F$ test of the analysis variance and the coefficient of variation (CV) of soil chemical attributes for the four management systems and in the three soil layers.

\begin{tabular}{|c|c|c|c|c|}
\hline \multirow[b]{2}{*}{ Atributos do solo } & \multicolumn{3}{|c|}{ Probabilidade do teste F } & \multirow{2}{*}{$\begin{array}{l}\text { CV } \\
(\%)\end{array}$} \\
\hline & $\begin{array}{l}\text { Manejo } \\
\text { (M) }\end{array}$ & $\begin{array}{c}\text { Camada } \\
\text { (C) }\end{array}$ & $\begin{array}{c}\text { Interação } \\
(\mathrm{M} \times \mathrm{C})\end{array}$ & \\
\hline COT (\%) & $0,000^{*}$ & $0,000^{*}$ & $0,025^{*}$ & 29,71 \\
\hline $\mathrm{pH}_{\left(\mathrm{H}_{2} \mathrm{O}\right)}$ & $0,000^{*}$ & $0,008^{*}$ & 0,091 & 3,47 \\
\hline $\mathrm{Al}+\mathrm{H}\left(\mathrm{cmol}_{\mathrm{c}} \mathrm{dm}^{-3}\right)$ & $0,000^{*}$ & $0,000^{*}$ & $0,001^{*}$ & 13,67 \\
\hline$P\left(\mathrm{mg} \mathrm{dm}^{-3}\right)$ & $0,000^{*}$ & $0,000^{*}$ & $0,000^{*}$ & 48,42 \\
\hline $\mathrm{K}\left(\mathrm{mg} \mathrm{dm}^{-3}\right)$ & $0,000^{*}$ & 0,159 & 0,886 & 28,56 \\
\hline $\mathrm{Ca}\left(\mathrm{cmol}_{\mathrm{c}} \mathrm{dm}^{-3}\right)$ & $0,000^{*}$ & 0,311 & $0,030^{*}$ & 15,58 \\
\hline $\mathrm{Mg}\left(\mathrm{cmol}_{\mathrm{c}} \mathrm{dm}^{-3}\right)$ & $0,000^{*}$ & 0,369 & 0,956 & 11,45 \\
\hline $\mathrm{Na}\left(\mathrm{mg} \mathrm{dm}^{-3}\right)$ & $0,000^{*}$ & $0,005^{\star}$ & 0,236 & 31,79 \\
\hline $\mathrm{Cu}\left(\mathrm{mg} \mathrm{dm} \mathrm{m}^{-3}\right)$ & $0,000^{*}$ & 0,988 & $0,002^{*}$ & 13,53 \\
\hline $\mathrm{Zn}\left(\mathrm{mg} \mathrm{dm}^{-3}\right)$ & $0,000^{*}$ & $0,000^{*}$ & $0,000^{*}$ & 26,55 \\
\hline $\mathrm{CTC}_{\text {efetiva }}\left(\mathrm{cmol}_{\mathrm{c}} \mathrm{dm}^{-3}\right)$ & $0,000^{*}$ & 0,412 & 0,115 & 13,32 \\
\hline $\mathrm{CTC}_{\mathrm{pH} 7,0}\left(\mathrm{cmol}_{\mathrm{c}} \mathrm{dm}^{-3}\right)$ & $0,000^{*}$ & 0,074 & 0,281 & 11,08 \\
\hline Saturação por bases (\%) & $0,000^{*}$ & $0,012^{*}$ & $0,022^{*}$ & 3,75 \\
\hline
\end{tabular}

${ }^{*}$ Teste F significativo $(p \leq 0,05)$.

Os teores de Zn no solo foram maiores na área de PP16, com diferença significativa em relação às áreas de PP4, PD e MN somente na camada superficial do solo (Figura 1B). Nesta camada, o incremento de Zn na área de PP16 foi de 3,2 vezes em relação à área de MN, indicando contribuição significativa dos DLS 
no aumento do elemento no solo. Maior concentração do micronutriente na camada superficial do solo também foi verificada por SCHERER et al. (2010) em três tipos de solos (Latossolo, Cambissolo e Neossolo) no estado de Santa Catarina em áreas de até 25 anos de uso de DLS e por CASSOL et al. (2011) e VEIGA et al. (2012) em Latossolo Vermelho com aplicações contínuas de doses crescentes de DLS (50, 100 e 200 $\mathrm{m}^{3} \mathrm{ha}^{-1}$ ) em seis e nove anos, respectivamente.
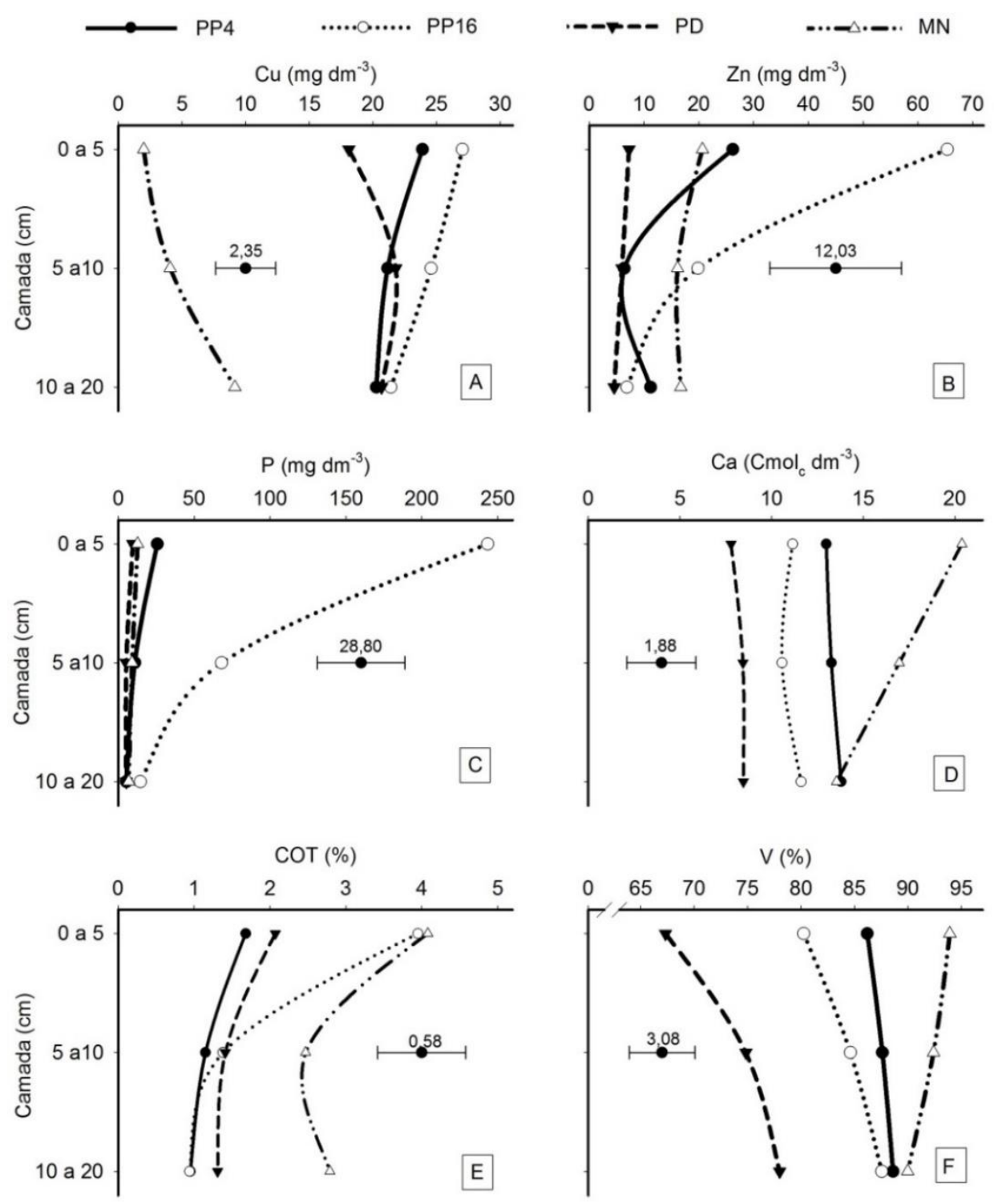

Figura 1. Indicadores químicos de qualidade do solo: $\mathrm{Cu}(\mathrm{A})$; Zn (B); P (C); Ca (D); carbono orgânico total, COT $(E)$ e saturação por bases, $V(F)$ nas áreas de pastagem perene de tifton com uso sucessivo de dejetos líquido de suínos durante quatro anos (PP4) e 16 anos (PP16), cultivo anual de grãos em plantio direto durante 16 anos (PD) e área de mata nativa (MN) em quatro camadas de solo. As barras horizontais indicam a diferença mínima significativa pelo teste $t$ de Student $(p \leq 0,05)$.

Figure 1. Chemical indicators of soil quality: $C u(A) ; Z n(B) ; P(C) ; C a(D) ;$ total organic carbon, TOC (E) and base saturation, $V(F)$ in the areas perennial Tifton pasture with successive use of liquid manure for 4 years (PP4) and 16 years (PP16), annual crop grain in no-till farming for 16 years (NTF) and native forest area (NF) in four soil layers. Horizontal bars indicate the least significant difference given by Student's t-test $(p \leq 0.05)$.

O intemperismo dos minerais presentes nas rochas ígneas extrusivas, como o basalto, comum da região sul do Brasil, também condiciona naturalmente para elevar os teores de $\mathrm{Cu}$ e $\mathrm{Zn}$ no solo (HUGEN et al. 2013). Os mesmos autores encontraram concentrações totais de $225 \mathrm{mg} \mathrm{kg}^{-1}$ de Cu e $92 \mathrm{mg} \mathrm{kg}^{-1} \mathrm{de} \mathrm{Zn}$ para o Neossolo derivado do basalto, semelhante ao solo do presente estudo. No caso do $\mathrm{Cu}$, os teores totais naturais encontrados já estão acima do limite de prevenção preconizado como valores orientadores de qualidade do solo pela Resolução $\mathrm{n}^{\circ} 420$ que são de $60 \mathrm{mg} \mathrm{kg}^{-1}$ (CONAMA 2009). Assim, na análise dos 
teores totais no solo deve-se considerar o tipo de material de origem, para não atribuir os altos valores à adição de DLS.

No que se refere a qualidade ambiental, TAVARES et al. (2013) orientam que os valores de referência devem ser definidos em cada estado brasileiro, em decorrência da variação dos teores naturais destes elementos nos solos. Além disso, valores de referência com bases nos teores disponíveis também poderia ser incluído como mais um parâmetro de referência para a qualidade do solo. A CQFS - RS/SC (2004) delimita que teores disponíveis no solo acima de 0,4 e 0,5 $\mathrm{mg} \mathrm{dm}^{-3}$ de $\mathrm{Cu}$ e $\mathrm{Zn}$, respectivamente, são considerados altos, mas não indica valores que podem comprometer a nutrição das plantas e a qualidade ambiental.

Os teores de $\mathrm{P}$ extraíveis no solo pelo método Mehlich ${ }^{-1}$ foram diferentes entre as áreas, destacando a PP16 com os maiores valores nas camadas de 0-5 e 5-10 cm (Figura 1C). Nesta área e na camada de 0-5 cm, o teor de $P$ foi 9,5, 25,6 e 19,2 vezes maior em relação à área de PP4, PD e MN, respectivamente. Nas áreas de tifton observam-se aumentos dos teores de $P$ na camada superficial do solo com o tempo de uso de DLS, resultados semelhantes aos relatados na maioria dos trabalhos com o uso de DLS (BERWANGER et al. 2008, GUARDINI et al. 2012, SCHERER et al. 2010), porém com distribuição variável do nutriente no perfil do solo com a dose, frequência de aplicação e tipo de solo. Em solos com menor estágio de intemperização (Neossolo) o deslocamento de $\mathrm{P}$ em profundidade pode chegar até $50 \mathrm{~cm}$ de profundidade após 15 anos de uso de DLS (SCHERER et al. 2010), pois há menos sítios de adsorção específica de argilominerais 1:1 e de óxidos de ferro e alumínio da fração argila em relação aos solos em estágio mais avançado de intemperização (Latossolo) (GUARDINI et al. 2012).

$\mathrm{O}$ deslocamento de $\mathrm{P}$ no solo ocorre à medida que os sítios de adsorção são saturados com ânions e está associado às áreas que recebem altas doses de DLS e com alta frequência de aplicação (GATIBONI et al. 2014). O aumento do carbono orgânico na camada superficial do solo, como observado na área de PP16 (Figura 1E) também pode ter contribuído para o deslocamento do $\mathrm{P}$ para a camada de $5-10 \mathrm{~cm}$, pois ocorre diminuição da capacidade máxima de adsorção de $\mathrm{P}$ nos colóides devido à competição por sítios de adsorção provenientes dos ânions da decomposição do carbono orgânico (BERWANGER et al. 2008). O deslocamento de $\mathrm{P}$ no perfil do solo é vantajoso, desde que o nutriente não ultrapasse o alcance das raízes e não comprometa à contaminação das águas subsuperficiais (HOUNTIN et al. 2000), pois pode estimular o crescimento do sistema radicular em profundidade e aumentar a demanda de água em períodos de estiagem (PAULETTI et al. 2014).

O teor de P no solo na área de PP16, na camada de 0-10 cm (média das camadas de 0-5 e 5-10 cm), foi de $155,59 \mathrm{mg} \mathrm{dm}^{-3}$, valor considerado "muito alto" pela CQFS - RS/SC (2004). Entretanto, nas áreas de PP4, PD e MN os valores não chegaram ao limite crítico, que é de $21 \mathrm{mg} \mathrm{kg}^{-1}$ para solos com teores de argila entre 210 a $400 \mathrm{~g} \mathrm{dm}^{-3}$ (CQFS - RS/SC 2004). Isto indica que a produção de tifton pode ser prejudicada nos primeiros anos com uso exclusivo de adubação com DLS e que a adubação mineral fosfatada utilizada para as culturas anuais na área PD durante 16 anos não foi adequada.

O valor "muito alto" na área de PP16 do ponto de vista de fertilidade do solo é adequado, mas o acúmulo de P na superfície do solo, predominantemente na forma orgânica, em área de aplicação na superfície e sem incorporação ao solo, pode aumentar o potencial de perdas por escoamento superficial (CERETTA et al. 2010, GIROTTO et al. 2010), comprometer a qualidade de águas superficiais pelo excesso do nutriente (GUARDINI et al. 2012) e eutrofização dos mananciais de água pelo crescimento excessivo de algas e plantas (KLEIN \& AGNE 2012). O desafio é delimitar doses de DLS que possibilitam o aumento dos teores de $P$ no solo, inclusive em profundidade, mas que não ultrapassem os limites pré-estabelecidos ou que não cause risco de contaminação ambiental.

Limites de $120 \mathrm{mg} \mathrm{kg}^{-1}$ de $\mathrm{P}$, usando o extrator Mehlich-1, são considerados críticos no solo para as condições de solo e clima nos EUA e a adubação fosfatada é suspensa para evitar riscos de contaminação ambiental (SHARPLEY et al. 1996). No Brasil, a legislação não prevê níveis críticos de P total ou disponível no solo, somente de P total na água (CONAMA 2005). No estado de Santa Catarina, GATIBONI et al. (2014) elaboraram uma proposta para os limites críticos ambientais de fósforo (LCA-P) na camada de 0-10 cm calculados pela equação: LCA-P $\left(\mathrm{mg} \mathrm{dm}^{-3}\right)=40+\%$ argila. Com base nesta equação, os teores de $P$ disponíveis no solo podem chegar até 60,$8 ; 70,1$ e $83,3 \mathrm{mg} \mathrm{dm}^{-3}$ na camada de 0-10 cm nas áreas de PP4, PP16 e PD, respectivamente. Estes valores evidenciam que a área de PP16 já ultrapassou o limite de $20 \%$ proposto por GATIBONI et al. (2014) e neste caso deve-se suspender imediatamente o uso de DLS e trabalhar com medidas mitigatórias imediatas para diminuir o risco de poluição ambiental. Entre estas medidas pode ser o revolvimento do solo para diluição do $\mathrm{P}$ no perfil do solo (associadas com medidas para evitar a erosão) (GATIBONI et al. 2014) e a inserção da cultivar Jiggs para maior extração e exportação do P do solo, que apresenta maior produção de biomassa vegetal em relação às cultivares de tifton (VENDRAMINI et al. 2010). 
Os valores de COT foram maiores na camada superficial do solo e mais expressivos na MN e PP16 (Figura 1E). Na área de MN ocorreu menor variação do COT nas três camadas de solo, com valores de 4,1\% na camada de $0-5 \mathrm{~cm}$ e 2,8\% na camada de $10-20 \mathrm{~cm}$, em relação a área de PP16, onde houve um forte gradiente, com decréscimo de 3,9\% na camada $0-5 \mathrm{~cm}$ à $0,9 \%$ na camada de $10-20 \mathrm{~cm}$. Com uso de DLS durante quatro anos em pastagem natural CERETTA et al. (2003) e ARRUDA et al. (2010) observaram variação no teor de COT em profundidade, com valores maiores na camada de $0-5 \mathrm{~cm}$, provavelmente devido ao aporte de matéria orgânica proveniente dos DLS. Na MN, os maiores valores de COT em todas as profundidades está relacionado ao aporte natural de resíduos orgânicos (serapilheira) (ROVEDDER et al. 2013).

O aumento do COT na camada superficial do solo na área de PP16 a valores próximos à da MN é um parâmetro positivo para a recuperação da qualidade do solo. VEZZANI e MIELNICZUK (2009) destacam que o COT é o principal atributo para avaliar a qualidade do solo. No entanto, o aumento do COT com o uso de DLS na área de PP16 está associado ao incremento dos teores de P acima do LCA-P, o que não é adequado do ponto de vista ambiental. O monitoramento das áreas de aplicação de DLS até atingir $80 \%$ do LCA-P e a adequação das doses a partir deste valor com base na quantidade de $\mathrm{P}$ exportada pela forrageira, acrescido do manejo do pastejo que proporciona maior aporte de material orgânico no solo, pode ser uma alternativa que associa a manutenção ou acréscimo de COT no solo sem comprometer a produção de forragem e a qualidade ambiental.

$\mathrm{O} \mathrm{pH}$, a CTC potencial e os teores de $\mathrm{Na}, \mathrm{K}$ e Mg trocáveis foram os atributos químicos do solo que não apresentaram interação significativa entre os sistemas de manejo e as camadas de solo (Tabela 2). $O$ $\mathrm{pH}$ do solo foi maior na MN, com valor de 6,9, seguido de 6,1, 6,0 e 5,6 nas áreas de PP16, PP4 e PD, respectivamente (Tabela 3). A substituição da cobertura florestal da $\mathrm{MN}$ pelos sistemas de produção de forragem (PP4 e PP16) e de culturas anuais de grãos (PD) provavelmente contribuíram para a acidificação do solo devido ao aumento da mineralização da matéria orgânica (ROVEDDER et al. 2013), acrescido da acidificação pelas reações de nitrificação proveniente dos fertilizantes nitrogenadas amoniacais (CRAVO et al. 2012) aplicados na área de cultivo anual de grãos (PD). Nas áreas de cultivo de tifton com uso de DLS (PP4 e PP16) o pH do solo foi mais próximo à $\mathrm{MN}$, que provavelmente está relacionado aos efeitos alcalinizantes do DLS (CHANTIGNY et al. 2004) e a complexação do alumínio trocável e a adsorção de íons $\mathrm{H}^{+}$na fração húmica do solo devido à alta reatividade dos ácidos fúlvicos e húmicos (CERETTA et al. 2003).

Tabela 3. Indicadores químicos de qualidade do solo nos quatro sistemas de manejo e nas três camadas de solo.

Table 3. Chemical indicators soil quality in the four management systems and in the three soil layers.

\begin{tabular}{|c|c|c|c|c|c|}
\hline \multirow{2}{*}{$\begin{array}{l}\text { Sistema de manejo e camada } \\
\text { de solo }{ }^{(1)}\end{array}$} & \multirow{2}{*}{$\mathrm{pH}_{\left(\mathrm{H}_{2} \mathrm{O}\right)}$} & $\mathrm{Na}$ & $\mathrm{K}$ & $\mathrm{Mg}$ & $\mathrm{CTC}_{\mathrm{pH} 7,0}$ \\
\hline & & \multicolumn{2}{|c|}{---- $\mathrm{mg} \mathrm{dm}^{-3}$---- } & \multicolumn{2}{|c|}{---- cmolc $\mathrm{dm}^{-3}$} \\
\hline & \multicolumn{5}{|c|}{----------------- Sistemas de manejo ----------------- } \\
\hline PP16 & $6,00 b^{(2)}$ & $34,33 \mathrm{a}$ & $1.044,33 \mathrm{a}$ & $4,55 \mathrm{a}$ & $21,93 \mathrm{a}$ \\
\hline PP4 & $6,10 \mathrm{~b}$ & $36,46 \mathrm{a}$ & $179,30 \mathrm{c}$ & $4,50 \mathrm{a}$ & $21,06 \mathrm{a}$ \\
\hline PD & $5,60 \mathrm{c}$ & $5,14 b$ & $58,83 d$ & $3,15 b$ & $15,74 \mathrm{~b}$ \\
\hline \multirow[t]{2}{*}{ MN } & $6,90 \mathrm{a}$ & $6,59 \mathrm{~b}$ & $329,8 \mathrm{~b}$ & $3,27 b$ & $22,85 \mathrm{a}$ \\
\hline & \multicolumn{5}{|c|}{ 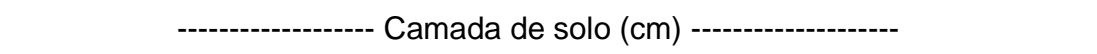 } \\
\hline $0-5$ & $5,97 \mathrm{~b}$ & $17,16 \mathrm{~b}$ & $447,35 \mathrm{a}$ & $3,75 \mathrm{a}$ & $21,62 \mathrm{a}$ \\
\hline $5-10$ & $6,23 a$ & $20,58 a b$ & $407,78 \mathrm{a}$ & $3,84 a$ & $20,12 a b$ \\
\hline $10-20$ & $6,24 \mathrm{a}$ & $24,14 \mathrm{a}$ & $354,07 \mathrm{a}$ & $4,01 \mathrm{a}$ & $19,45 b$ \\
\hline
\end{tabular}

(1)PP4: pastagem perene de tifton com uso sucessivo de dejetos líquido de suínos durante quatro anos; PP16: pastagem perene de tifton com uso sucessivo de dejetos líquido de suínos durante 16 anos; PD: cultivo anual de grãos em plantio direto durante 16 anos; MN: mata nativa. ${ }^{(2)}$ Médias seguidas por letras distintas, na coluna, diferem entre si pelo teste t de Student $(p \leq 0,05)$.

Os teores de Na foram mais elevados nas áreas com adição de DLS (PP4 e PP16), sendo 6,0 vezes maior que nas áreas MN e PD (Tabela 3). ASSEFA et al. (2004) observaram que o uso de resíduos orgânicos de origem animal tendem a aumentar significativamente os teores de $\mathrm{Na}$ no solo, como foi verificado neste trabalho. Isso pode ocorrer por causa do alto teor deste elemento adicionado na ração dos animais, resultando em maior salinidade do DLS e, consequentemente, de seu acúmulo no solo (LI-XIAN et al. 2007). Este aumento pode causar efeitos negativos no solo, pois incrementa os teores de cátions e com isso compromete 
as propriedades físico-hídricas do solo e o crescimento das plantas (MATOS et al. 2014). Semelhante ao Na, os teores de Mg também foram maiores nas áreas de adição de DLS, mas com menor incremento (1,4 vezes) em relação a média das áreas de $\mathrm{MN}$ e PD. Destaca-se que os teores de $\mathrm{Mg}$ são altos nas condições naturais do solo $(\mathrm{MN})$ e que o cultivo de grãos $(\mathrm{PD})$ não reduziu o teor no solo, possivelmente devido à aplicação de calcário dolomítico para correção da acidez do solo.

A área de PP16 apresentou incremento no teor de $\mathrm{K}$ no solo $\left(1.044,33 \mathrm{mg} \mathrm{dm}^{-3}\right)$, seguido pelas áreas MN, PP4 e PD, com 329,8; 179,3 e 58,83 $\mathrm{mg} \mathrm{dm}^{-3}$, respectivamente (Tabela 3). CASSOL et al. (2011) observaram, em trabalho semelhante, que com seis anos de aplicações de DLS o teor de $\mathrm{K}$ extraível na camada mais superficial do solo aumentou em até 1,8 e 3,3 vezes, com 50 e $200 \mathrm{~m}^{3} \mathrm{ha}^{-1}$, respectivamente. O teor de $\mathrm{K}$ da área de MN foi 11,6 vezes maior que o limite crítico estabelecido pela CQFS - RS/SC (2004) que é de $90 \mathrm{mg} \mathrm{dm}^{-3}$ para solos com CTC potencial maior que $15 \mathrm{cmol}_{\mathrm{c}} \mathrm{dm}^{-3}$. Observa-se que na área PP16 foi adicionado uma quantidade de $\mathrm{K}$ além da necessidade da forrageira com uso de $150 \mathrm{~m}^{3} \mathrm{ha}^{-1}$ de DLS durante 16 anos no cultivo de tifton, apesar de ser uma forrageira de alta extração de K (SARMENTO et al. 2006), indicando que precisa redefinir a dose e a frequência de aplicação de DLS. Ao contrário, o teor de $K$ no solo na área de PD foi menor que a $\mathrm{MN}$ e menor que o limite crítico, indicando que a adubação mineral potássica nas culturas de grãos foi aquém da necessidade das culturas e provavelmente está comprometendo a produção de grãos.

O teor de $\mathrm{K}$ foi uniforme nas três camadas de solo, independentemente dos sistemas de manejo (Tabela 3). Esta homogeneidade de $\mathrm{K}$ no perfil do solo é parâmetro importante para o crescimento radicular em profundidade, mas devido à pequena profundidade efetiva do perfil (máximo de $79 \mathrm{~cm}$ ) (Tabela 1) pode ocorrer perdas do nutriente por lixiviação. Assim, a área de PP16 é mais vulnerável à perda de $\mathrm{K}$ pela frequência de adição de DLS, pois segundo SCHERER et al. (2010) o deslocamento e a distribuição de K no perfil do solo é intensificado pelos teores iniciais do nutriente. Isto reforça a necessidade de readequação de doses e frequência de aplicação de DLS.

A CTC potencial foi maior na MN, não diferindo das áreas de PP4 e PP16 (Tabela 3). Isto pode ser explicado pelo aumento do COT nas áreas de aplicação de DLS, principalmente na camada de 0-5 cm (Figura $1 \mathrm{E})$, pois a matéria orgânica contribui significativamente no aumento das cargas negativas do solo (BRIEDIS et al. 2012) e, consequentemente, aumenta a retenção dos cátions ( $\mathrm{Na}, \mathrm{K}, \mathrm{Ca}$ e $\mathrm{Mg}$ ) adicionados pelos DLS. Além disso, contribuiu para o aumento da saturação por bases, nas três camadas de solo (Figura 1F). A menor CTC na área de PD está relacionada à degradação do COT pelo sistema de cultivo, mesmo sem revolvimento do solo, indicando que o sistema de produção ainda não está contribuindo com adição de carbono suficiente para a manutenção da matéria orgânica próxima a área de $\mathrm{MN}$.

\section{CONCLUSÃO}

A CTC, o COT e os teores de $\mathrm{Cu}$, Zn e P do solo são atributos químicos que podem ser usados como indicadores de qualidade do solo.

Nas áreas de cultivo com tifton o uso de dejetos líquidos de suínos por 16 anos aumenta o teor de $\mathrm{P}$ no solo acima do limite crítico ambiental (LCA-P).

No sistema de cultivo de grãos com adubação mineral em plantio direto houve redução do carbono orgânico e da capacidade de troca de cátions em relação à mata nativa.

O uso de dejetos líquidos de suínos durante quatro ou 16 anos no cultivo de tifton contribuem para o aumento dos teores de Cu e Zn na camada superficial do solo em comparação com a MN.

\section{REFERÊNCIAS}

ANAMI MH et al. 2008. Deslocamento miscível de nitrato e fosfato proveniente de água residuária da suinocultura em colunas de solo. Revista Brasileira de Engenharia Agrícola e Ambiental 12: 75-80.

ARRUDA CAO et al. 2010. Aplicação de dejeto suíno e estrutura de um Latossolo Vermelho sob semeadura direta. Ciência e Agrotecnologia 34: 804-809.

ASSEFA BA et al. 2004. Effects of four annual applications of manure on black Chernozemic soils. Canadian Biosystems Engineering 46: 39-46.

BARBOSA GC et al. 2009. Produção de massa seca de aveia branca em solo com aplicação de resíduos animais. Synergismus scyentifica 4: 1-3.

BERWANGER AL et al. 2008. Alterações no teor de fósforo no solo com aplicação de dejetos líquidos de suínos. Revista Brasileira de Ciência do Solo 32: 2525-2532.

BRIEDIS C et al. 2012. Carbono do solo e atributos de fertilidade em resposta à calagem superficial em plantio direto.

Pesquisa Agropecuária Brasileira 47: 1007-1014.

CASSOL PC et al. 2011. Atributos químicos em Latossolo Vermelho fertilizado com dejeto suíno e adubo solúvel. Revista 
de Ciências Agroveterinárias 2: 103-112.

CASSOL PC et al. 2012. Disponibilidade de macronutrientes e rendimento de milho em Latossolo fertilizado com dejeto suíno. Revista Brasileira de Ciência do Solo 36: 1911-1923.

CERETTA CA et al. 2003. Características químicas de solo sob aplicação de dejeto líquido de suínos em pastagem natural. Pesquisa Agropecuária Brasileira 38: 729-735.

CERETTA CA et al. 2010. Frações de fósforo no solo após sucessivas aplicações de dejetos de suínos em plantio direto. Pesquisa Agropecuária Brasileira 45: 593-602.

CHANTIGNY MH et al. 2004. Ammonia volatilization and selected soil characteristics following application of anaerobically digested pig slurry. Soil Science Society of America Journal 68: 306-312.

CONAMA. 2005. Conselho Nacional do Meio Ambiente. Resolução no 359, de 29 de abril de 2005. Ministério do Meio Ambiente. Diário Oficial da República Federativa do Brasil, Brasília, DF, no 083, 3 maio. Seção 1, p.63-64.

CONAMA. 2009. Conselho Nacional do Meio Ambiente. Resolução no 420, de 28 de dezembro de 2009. Ministério do Meio Ambiente. Diário Oficial da República Federativa do Brasil, Brasília, DF, n.249, 7 dez. Seção 1, p.81-84.

CQFS-RS/SC 2004. Comissão de Química e Fertilidade do Solo RS/SC. Manual de adubação e calagem. Porto Alegre: SBCS. 400p.

CRAVO MS et al. 2012. Calagem em Latossolo Amarelo distrófico da Amazônia e sua influência em atributos químicos do solo e na produtividade de culturas anuais. Revista Brasileira de Ciência do Solo 36: 895-907.

DONAGEMA GK et al. 2011. Manual de métodos de análise de solo. Rio de Janeiro: Embrapa Solos. 230p.

GATIBONI LC et al. 2014. Proposta de limites críticos ambientais de fósforo para solos de Santa Catarina. Lages: CAV/UDESC. 38p. (Boletim Técnico).

GIROTTO E et al. 2010. Acúmulo e formas de cobre e zinco no solo após aplicações sucessivas de dejeto líquido de suínos. Revista Brasileira Ciência do Solo 34: 955-965.

GUARDINI R et al. 2012. Phosphorus accumulation and pollution potential in a hapludult fertilized with pig manure. Revista Brasileira de Ciência do Solo 36: 1333-1342.

HOUNTIN JA et al. 2000. Use of a fractionation procedure to assess the potential for phosphorus movement in a soil profile after 14 years of liquid pig manure fertilization. Agriculture, Ecosystems \& Environment 78: 77-84.

HUGEN C et al. 2013. Teores de Cu e Zn em perfis de solos de diferentes litologias em Santa Catarina. Revista Brasileira de Engenharia Agrícola e Ambiental 17: 622-628.

KLEIN C \& AGNE SAA. 2012. Fósforo: de nutriente à poluente! Revista Eletrônica em Gestão, Educação e Tecnologia Ambiental 8: 1713-1721.

LI-XIAN Y et al. 2007. Salinity of animal manure and potential risk of secondary soil salinization through successive manure application. Science of the Total Environment 383: 106-114.

MAGGI CF et al. 2013. Impacto da aplicação de água residuária de suinocultura no percolado e no solo cultivado com soja. Revista Engenharia Agrícola 33: 279-290.

MALUF JRT 2000. Nova classificação climática do Estado do Rio Grande do Sul. Revista Brasileira de Agrometeorologia 8: 141-150.

MATOS AT et al. 2014. Saturação do complexo de troca de solos oxídicos com sódio. Revista Brasileira de Engenharia Agrícola e Ambiental 18: 501-506.

MOREIRA A et al. 2009. Extratores e disponibilidade de micronutrientes em Terra Preta de Índio da Amazônia Central. Ciencia del Suelo 27: 127-134.

PAULETTI V et al. 2014. Efeitos em longo prazo da aplicação de gesso e calcário no sistema de plantio direto. Revista Brasileira de Ciência do Solo 38: 495-505.

ROVEDDER APM et al. 2013. Compreensão e aplicabilidade do conceito de solo florestal. Ciência Florestal 23: 517-528. SANTOS HG et al. 2013. Sistema brasileiro de classificação de solos. Brasília: Embrapa. 353p.

SARMENTO P et al. 2006. Nutrientes limitantes ao desenvolvimento do capim-tifton 85 em Argissolo Vermelho-Amarelo. Boletim de Indústria Animal 63: 11-18.

SARTOR LR et al. 2012. Effect of swine residue rates on corn, common bean, soybean and wheat yield. Revista Brasileira de Ciência do Solo 36: 661-669.

SCHERER EE et al. 2010. Atributos químicos do solo influenciados por sucessivas aplicações de dejetos suínos em áreas agrícolas de Santa Catarina. Revista Brasileira de Ciência do Solo 34: 1375-1383.

SHARPLEY A et al. 1996. Determining environmentally sound soil phosphorus levels. Journal of Soil and Water Conservation 51: 160-166.

STEINER F et al. 2011. Disponibilidade de micronutrientes no sistema plantio direto, na presença e ausência de plantas de cobertura submetido a diferentes fontes de fertilizantes. Global Science and Technology 4: 28-37.

TAVARES SRL et al. 2013. Avaliação de espécies vegetais na fitorremediação de solos contaminados por metais pesados. Holos 5: 80-97.

TEDESCO MJ et al. 1995. Análise de solo, plantas e outros materiais. Porto Alegre: UFRGS. 174p.

TIECHER TL et al. 2013. Forms and accumulation of copper and zinc in a Sandy Typic Hapludalf soil after long-term application of pig slurry and deep litter. Revista Brasileira de Ciência do Solo 37: 812-824.

VENDRAMINI JMB et al. 2010. Nutritive value and fermentation parameters of warm-season grass silage. The Professional Animal Scientist 2: 193-200.

VEIGA M et al. 2012. Chemical attributes of a Hapludox soil after nine years of pig slurry application. Pesquisa Agropecuária Brasileira 47: 1766-1773. 
Pessotto et al.

VEZZANI FM \& MIELNICZUK J. 2009. Uma visão sobre qualidade do solo. Revista Brasileira de Ciência do Solo 33: 743755.

YEOMANS JC \& BREMNER JM. 1988. A rapid and precise method for routine determination of organic carbon in soil. Communications in Soil Science and Plant Analysis 19: 1467-1476. 